\title{
Performance and Acceptability of Two Self-Inflating Bag-Mask Neonatal Resuscitator Designs
}

\author{
Patricia S Coffey PhD MPH, Eugene A Saxon MME MEng, Indira Narayanan MD, and \\ Robert M DiBlasi RRT-NPS FAARC
}

\begin{abstract}
BACKGROUND: A self-inflating bag-mask device is specified by international policy guidelines as standard prototype of care for newborn resuscitation. Our hypothesis is that a new bag-mask design would be as effective and easy to use as a standard, self-inflating resuscitation bag-mask. METHODS: We conducted a comparative evaluation of the performance and acceptability of the Laerdal 220-mL resuscitator with a size-1 mask (NeoNatalie) and a Laerdal prototype Upright resuscitator with a modified mask. Participants evaluated the devices in random order using a commercially available test lung and training mannikin with an integrated chest-rise module. The test lung was configured with healthy and sick newborn lung mechanics. Two user groups participated: (1) frequent users who had used manual resuscitators to resuscitate infants and (2) infrequent users who received competency-based training and had not previously used manual resuscitators to resuscitate infants. RESULTS: Thirty-eight individuals participated in the study during March 2013. Both resuscitators are capable of delivering the minimum required tidal volumes to newborns. The Upright device provided a significant reduction in the percentage of inadequate ventilations $(<12.5 \mathrm{~mL}$ ) compared with the NeoNatalie. Although the test sequences with low-compliance lung settings showed no difference in the percentage of excessive ventilations $(>37.5 \mathrm{~mL}$ ) between the Upright and NeoNatalie, the test sequences with normal-compliance lung settings showed a higher percentage of excessive ventilations with both, and the increase was greater with the Upright than with the NeoNatalie $(85.92 \%$ vs $71.39 \%, P<.001)$. The subjective acceptability and disassembly/ reassembly tests were supportive of the new device design. CONCLUSIONS: The performance and acceptability of the Upright device in this user population suggest that the device may be suitable for effective ventilation by infrequent users in low-resource settings. The Upright device should be tested in such a setting. Key words: ventilation; medical devices; evaluation; newborn asphyxia; adverse intrapartum events; neonatal resuscitation. [Respir Care 2015;60(9):1227-1237. (c) 2015 Daedalus Enterprises]
\end{abstract}

\section{Introduction}

Nearly $50 \%$ of all newborn deaths occur within the first $24 \mathrm{~h}$ after birth, with the majority resulting from adverse intrapartum events, including birth asphyxia. This condi-

Drs Coffey and Narayanan and Mr Saxon are affiliated with PATH, Seattle, Washington. Mr DiBlasi is affiliated with the Department of Respiratory Therapy, Seattle Children's Hospital, and the Center for Developmental Therapeutics, Seattle Children's Hospital Research Institute, Seattle, Washington.

Laerdal Global Health funded this study as an independent evaluation of their devices. Mr DiBlasi has disclosed relationships with Dräger, Ikaria, and Monaghan Medical. tion, manifesting as the failure of a newborn to establish adequate breathing after birth, affects 717,000 newborns each year, accounting for almost one quarter of newborn deaths worldwide. ${ }^{1}$ Additionally, there are an estimated 2.65 million stillbirths every year, an unknown number of which may be live-born but misclassified as fresh stillbirth when no resuscitation has been provided. ${ }^{2}$ A number of these deaths could easily be prevented with basic neonatal

\footnotetext{
Correspondence: Patricia S Coffey PhD MPH, PATH, 2201 Westlake Avenue, Suite 200, PO Box 900922, Seattle, WA 98109. E-mail: pcoffey@path.org.
}

DOI: $10.4187 /$ respcare. 03867 
resuscitation by a skilled birth attendant. Capacity building of such health workers can be achieved through competency-based training. For many babies born in low-resource settings, however, this basic intervention is not available. Ensuring universal access to newborn resuscitation is an essential and necessary challenge to reduce neonatal mortality.

\section{See the Related Editorial on Page 1367}

A self-inflating bag-mask device is specified by international policy guidelines as standard, evidence-based technology for newborn resuscitation. ${ }^{3}$ It has, in addition, a particular advantage in low-resource settings, which often do not have the blended air/oxygen gas source necessary to operate flow-inflating bags and T-piece devices. ${ }^{4}$ Despite advances in features and materials, the bag-mask technology is not new. The first manual resuscitation device with a self-inflating bag was developed in $1954 . .^{5}$ The current innovation in the product category focuses on simplification of device design and parts. This may make it particularly useful with infrequent users at peripheral health centers who may be better able to embrace new technologies. To understand this further, we conducted a study to determine whether there are differences in the performance and acceptability between a commonly used infant manual resuscitator and a new resuscitator design.

\section{Methods}

\section{Study Population and Design}

The study was a comparative evaluation of the performance and acceptability of 2 resuscitator designs: the Laerdal 220-mL resuscitator with a size-1 mask (also known as NeoNatalie, Laerdal Global Health, Stavanger, Norway) and a prototype self-inflating bag-mask (Upright resuscitator with a modified mask, Laerdal Global Health). The devices were evaluated by participants in random order using a commercially available test lung (ASL 5000, IngMar Medical, Pittsburgh, Pennsylvania) and training mannikin (NeoNatalie, Laerdal Global Health) with an integrated chest-rise module. Sample size was estimated based on pilot data previously collected (Laerdal Global Health, unpublished data) on the proportion of adequate ventilations when using the NeoNatalie resuscitator versus the Upright resuscitator in a lung model with normal lung compliance. Two user groups participated in this evaluation: (1) frequent users who had used manual resuscitators on babies (respiratory therapists, neonatologists, and neonatal ICU nurses) and were currently working at Seattle Children's Hospital and (2) infrequent users who had received competency-based training but had never used a

\section{QUICK LOOK}

\section{Current knowledge}

A self-inflating bag-mask resuscitator is specified by international policy guidelines as standard, evidencebased technology for newborn resuscitation. Neonatal resuscitators include self- and flow-inflating devices, the designs of which have not changed appreciably over the last few decades. Safe and effective use of a resuscitator requires adequate caregiver training and proper device design.

\section{What this paper contributes to our knowledge}

In a neonatal lung model, the larger-volume upright neonatal resuscitator reduced inadequate delivered tidal volumes $(<5 \mathrm{~mL} / \mathrm{kg})$ compared with a traditional resuscitator in the hands of inexperienced users. Excessive tidal volumes $(>15 \mathrm{~mL} / \mathrm{kg}$ ) were observed in some cases with both resuscitators, but more so with the upright resuscitator, regardless of clinician experience, and this was present only in the lung model when configured with normal compliance. The upright orientation and larger volume appear to counter volume loss due to leaks around the mask. Future studies may be needed to address outcomes in infants.

bag-mask with a baby. This group included respiratory therapy or midwifery students or midwives from local universities and/or midwifery practices. This study was approved by the Seattle Children's Hospital institutional review board (14096) and the PATH Research Ethics Committee (HS692).

\section{Resuscitation Devices}

For the purposes of this evaluation, the Laerdal NeoNatalie bag-mask resuscitator (Fig. 1A) was used as the reference comparison device for standard care. The NeoNatalie bag-mask costs $\$ 15$, is made of silicone and polysulfonate, can be boiled or autoclaved, is extremely durable, and comes with 2 mask sizes (0 and 1). For this evaluation, only the larger mask (size 1) was used because the objective was to assess device performance in term newborns and larger preterm/low-birthweight babies. The size-1 mask is designed to be used for babies in this category. The NeoNatalie resuscitator is composed of 9 parts (plus the mask) and a $220-\mathrm{mL}$ bag that is oriented horizontally and perpendicularly to the mask. The pressurerelief valve is adjacent to the patient valve.

The Upright design (Fig. 1B) is intended by the manufacturer to be less expensive and easier to use and clean by 


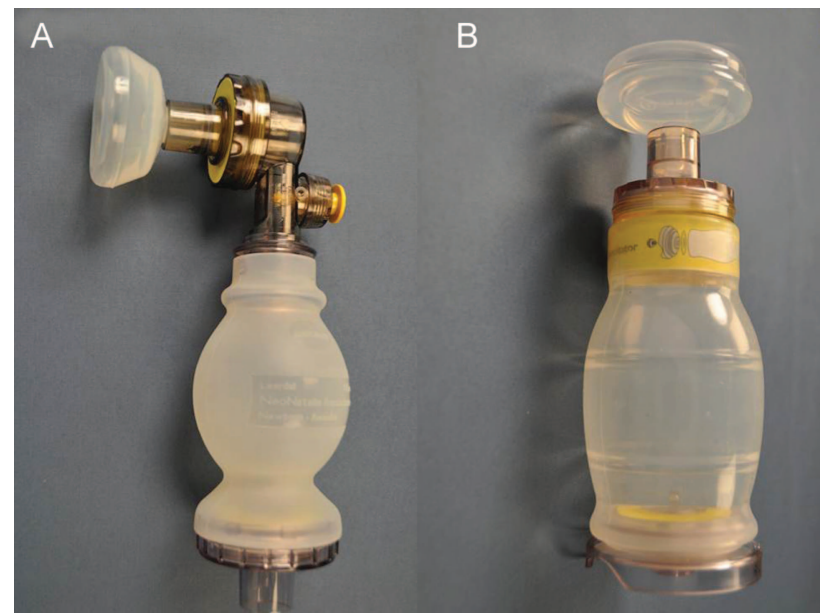

Fig. 1. A: NeoNatalie resuscitator. B: Upright resuscitator.

health workers. This new design also includes a modified mask that is thicker and less flexible in the non-contacting areas but with a thinner, pillow-like seal to provide a more reliable patient/mask interface. The mask also features a ridge on the stem that mates with a groove in the patient valve, providing for a more robust connection between the two. The Upright resuscitator is composed of fewer parts (6 plus the mask), has a larger bag volume of $320 \mathrm{~mL}$, and is oriented vertically in line with the mask. The Upright resuscitator bag has thicker walls, and the pressure-relief valve has been relocated to the distal end of the device in the center of the intake valve.

The International Organization for Standardization (ISO) specifies a pressure limit of $45 \mathrm{~cm} \mathrm{H}_{2} \mathrm{O}$ for devices intended for infants under $10 \mathrm{~kg} .{ }^{6}$ Although both of these devices are under the ISO limit, the Upright pressure-relief valve is set at a higher point than the NeoNatalie (Upright, $45 \mathrm{~cm} \mathrm{H}_{2} \mathrm{O}$; NeoNatalie, $35 \mathrm{~cm} \mathrm{H}_{2} \mathrm{O}$ ). Setting the pressure-relief limit for the Upright at the higher end of the ISO range is designed to allow increased ability to inflate the lungs of newborns with low compliance, as in the initial stages of birth asphyxia, and in preterm babies with surfactant deficiency and/or fluid-filled lungs.

\section{Study Procedures}

After obtaining informed consent from participants, demographic information and hand size were recorded (hand length and width measured using a centimeter grid). Participants were oriented to resuscitator designs and mask placement using a standardized video and then asked to disassemble each type of resuscitator to its primary components. The time used to disassemble each device was recorded. Written instructions for disassembly were not provided to assess the likelihood of human error in this task. Participants were then instructed to ventilate the Neo-
Natalie training mannikin (approximate simulated weight of 2-3 kg) for 4 min continuously with each device using the test sequence below (Table 1). The choice of $4 \mathrm{~min}$ was based on the mean duration of ventilation required to revive asphyxiated babies requiring face-mask ventilation in a low-resource country. ${ }^{7}$ The ASL 5000 test lung was configured to mimic the array of lung mechanics that an infant may experience in the first few minutes of life. Because newborn lung mechanics have not been described in the literature on postpartum resuscitation, we chose to alternate the lung mechanics (resistance and compliance) between normal-term newborn conditions and those previously described in preterm infants with respiratory distress syndrome. ${ }^{8}$

A specialized, real-time chest-rise module (IngMar Medical) inflated a bladder within the chest wall of the NeoNatalie infant mannikin with a volume of gas that was proportional to the pressure magnitude, duration, and frequency of the breaths provided to the ASL 5000. If a larger pressure was delivered to the lung model, then excessive chest rise could be observed. Conversely, when inadequate breaths were delivered (eg, poor mask seal or leak), then poor chest rise would be observed. As such, clinicians would need to vary support levels based on perceived changes in compliance, resistance, and chest rise.

All pressure and volume data from the 4-min epoch were obtained from within the test lung and translated from the ASL software data (version 3.2) into an Excel (Microsoft, Redmond, Washington) spreadsheet. We determined a priori excessive pressure delivery as any instances of pressure that exceeded $60 \mathrm{~cm} \mathrm{H}_{2} \mathrm{O}$ in the lung model. In general, an inflation pressure of $30 \mathrm{~cm} \mathrm{H}_{2} \mathrm{O}$ in term babies ${ }^{9-11}$ and $20-25 \mathrm{~cm} \mathrm{H}_{2} \mathrm{O}$ in preterm babies, ${ }^{12}$ when applied appropriately, is adequate to produce the necessary improvement in chest expansion and heart rate.

Assuming we were working with a neonate of $\sim 2-3 \mathrm{~kg}$ (NeoNatalie), we determined a priori the degree of tidal volume $\left(\mathrm{V}_{\mathrm{T}}\right)$ as the proportion of ventilations that were: (1) adequate (respiratory volume of $\geq 10 \mathrm{~mL}$, $>5 \mathrm{~mL} / \mathrm{kg}$ ), (2) inadequate (inspiratory volume of $<10$, $<5 \mathrm{~mL} / \mathrm{kg}$ ); and (3) excessive (inspiratory volume of $>30, \sim 15 \mathrm{~mL} / \mathrm{kg}$ ). Excessive ventilation was counted as part of adequate ventilation because it may allow ventilation of the lungs and thereby eliminate carbon dioxide from the lungs and deliver oxygen to the newborn. Frequencies of $<30,>40$, and $>60$ breaths/min were noted as well.

Following completion of ventilation sequences, participants were asked to reassemble each type of resuscitator. The time used to reassemble each device was recorded. Written instructions for reassembly were not provided to assess the likelihood of human error in this task. Each participant recorded observations about each device on data collection sheets using a 5-point Likert-type scale to 
Table 1. Simulation Conditions and Testing Epochs

\begin{tabular}{llclrr}
\hline \hline & \multicolumn{1}{c}{ Description } & Total Duration (min) & Ventilation Rate & Compliance $\left(\mathrm{mL} / \mathrm{cm} \mathrm{H}_{2} \mathrm{O}\right)$ & Resistance $\left(\mathrm{cm} \mathrm{H} \mathrm{H}_{2} \mathrm{O} / \mathrm{L} / \mathrm{s}\right)$ \\
\hline Step 1 & Low compliance & 1 & $30-40$ breaths/min & 0.5 & 160 \\
Step 2 & Prolonged inflations (5 breaths) & 1 & $2-3-\mathrm{s}$ inflations & 0.5 & 160 \\
Step 3 & Normal compliance & 2 & 30 to 40 breaths/min & 2.0 & 50
\end{tabular}

Simulated ventilation using the ASL 5000 (4 min)

assess variables related to device usability and selected their preferred device. At the end of the session, each participant was offered the opportunity to debrief with a researcher about his or her experience with the resuscitators, highlighting differences between the 2 designs, including issues with disassembly/reassembly, ergonomics, and correct use. Structured observation checklists were used by research staff during device disassembly, reassembly, and ventilation sequences. The resuscitation sequence required $\sim 40 \mathrm{~min}$ for both devices. During data collection, the average room temperature $\left(72.9 \pm 1.01^{\circ} \mathrm{F}\right)$, relative humidity $(34.3 \pm 4.70 \%)$, and pressure $(29.2 \pm-0.88 \mathrm{~mm} \mathrm{Hg})$ were relatively constant.

\section{Statistical Analysis}

Quantitative data from the test lung were reduced and analyzed using SPSS 12.0 (SPSS, Chicago, Illinois) and Stata 12.0 (StataCorp, College Station, Texas). Performance data for the ventilation sequences were sampled at $512 \mathrm{~Hz}$, and breath-by-breath data were downloaded from the ASL 5000. Qualitative data from the debriefing sessions were cleaned and manually sorted into thematic blocks of text. Device usability was measured as the mean of scores generated from a 5-point Likert-type scale for human factor variables related to ease of use, comfort, and ergonomics. A similar set of scores was generated for variables related to disassembly/reassembly. A summary device usability score was measured as the mean of all scores on the device usability index. Paired $t$ test and 2-tailed chi-square tests were used when comparing devices. Participant performance of disassembly and reassembly was compared using a 2-way analysis of variance. $P<.05$ was considered statistically significant. All data were stratified by 2 categorical variables: (1) frequent/infrequent users and (2) hand size. Hand-size cut-points were determined using Department of Trade and Industry standards, which are based on hand anthropometry data from the Georgia Tech Research Institute web site (http://usability.gtri.gatech.edu/ eou_info/hand_anthro.php, Accessed May 20, 2015).

\section{Results}

Thirty-eight individuals participated in the study during March 2013. All participants completed the simulated use and acceptability portions of the protocol, and 33 participants completed an additional component related to device disassembly/reassembly and the debriefing interview. Participants were primarily respiratory therapists (29\%), nurses or nurse midwives $(26 \%)$, and midwives (13\%), among others. The majority (74\%) of participants had used a resuscitation device to resuscitate a newborn. Demographic characteristics are reported in Table 2.

\section{Performance}

Lung model peak inspiratory pressure (PIP) was greater with the Upright resuscitator than with the NeoNatalie resuscitator under all testing conditions $(P<.05)$ for the 4-min testing epoch for both groups and for all hand sizes (Fig. 2). Pressures ranged from 19.27 to $41.86 \mathrm{~cm} \mathrm{H}_{2} \mathrm{O}$ for the Upright resuscitator and from 4.77 to $35.02 \mathrm{~cm} \mathrm{H}_{2} \mathrm{O}$ for the NeoNatalie resuscitator. Excessive PIP (\% over $60 \mathrm{~cm} \mathrm{H}_{2} \mathrm{O}$ ) was observed more frequently with the Upright resuscitator than with the NeoNatalie $(0.44 \%$ vs $0.19 \%, P<.02$ ) regardless of user experience level or hand size.

Lung model $\mathrm{V}_{\mathrm{T}}$ was greater overall with the Upright resuscitator than with the NeoNatalie resuscitator and for frequent users and all hand sizes $(P<.05)$, but was not different for infrequent users $(P=.056)$ during the 4-min testing epoch (Fig. 3). $\mathrm{V}_{\mathrm{T}}$ ranged from 9.0 to $54.5 \mathrm{~mL}$ for the Upright resuscitator and from 24.5 to $64.7 \mathrm{~mL}$ for the NeoNatalie resuscitator. Inadequate volume delivery to the lung model $(<5 \mathrm{~mL} / \mathrm{kg}, 12.5 \mathrm{~mL})$ was observed less frequently with the Upright resuscitator than with the NeoNatalie resuscitator $(6.2 \%$ vs $14.4 \%, P<.001)$. Excessive volume delivery to the lung model ( $>15 \mathrm{~mL} / \mathrm{kg}, 37.5 \mathrm{~mL}$ ) was observed more frequently with the Upright resuscitator compared with the NeoNatalie ( $43.9 \%$ vs $37.4 \%, P<.001$ ) regardless of user experience or hand size.

There were no differences in lung model breathing frequency between the 2 manual resuscitators $(P=.41)$ over the 4-min testing epoch regardless of user experience or 
Table 2. Participant Demographics

\begin{tabular}{|c|c|}
\hline Parameter & Values \\
\hline \multicolumn{2}{|l|}{ Sex } \\
\hline Females & $30(79.0)$ \\
\hline Males & $8(21.0)$ \\
\hline \multicolumn{2}{|l|}{ Professional designation* } \\
\hline Neonatologist & $4(10.5)$ \\
\hline Pediatrician & $3(7.9)$ \\
\hline Physician & $1(2.6)$ \\
\hline Respiratory therapist & $11(29.0)$ \\
\hline Nurse, nurse midwife & $10(26.3)$ \\
\hline Midwife & $5(13.2)$ \\
\hline Midwifery student & $2(5.3)$ \\
\hline Respiratory therapist student & $2(5.3)$ \\
\hline \multicolumn{2}{|c|}{ Neonatal resuscitator training completed in lifetime } \\
\hline No & $1(2.6)$ \\
\hline Yes & $37(97.4)$ \\
\hline \multicolumn{2}{|c|}{$\begin{array}{l}\text { Neonatal resuscitator training completed in } \\
\text { previous } 12 \mathrm{~m}\end{array}$} \\
\hline No & $16(42.1)$ \\
\hline Yes & $22(57.9)$ \\
\hline \multicolumn{2}{|c|}{ Ever used resuscitator to resuscitate newborn } \\
\hline No (infrequent users) & $10(26.3)$ \\
\hline Yes (frequent users) & $28(73.7)$ \\
\hline \multicolumn{2}{|l|}{ Hand size } \\
\hline Average and below average & $21(55.3)$ \\
\hline Above average & $17(44.7)$ \\
\hline
\end{tabular}

hand size (Fig. 4). Breathing frequency ranged from 0 to 62.8 breaths/min for the NeoNatalie and from 0 to 55.8 breaths/min for the Upright resuscitator. Breathing frequency percentage at $<30$ breaths/min was lower when subjects used the Upright resuscitator compared with the NeoNatalie ( $12 \%$ vs $23 \%, P=.01)$. There were no differences in breathing frequency percentage at $>60$ breaths/min between the NeoNatalie and Upright resuscitators (4\% vs $8 \%, P=.59$ ) over the 4-min testing epoch (see Fig. 4). Data for performance outcome variables for the 4-min testing epoch are displayed in Table 3.

During the ventilation sequences, 23 participants were observed to obtain chest rise for both devices. The absence of chest rise with all other participants ${ }^{13}$ was due to an equipment malfunction with the ASL 5000 chest-rise module. We compared performance measures of each device for the 4-min testing epoch stratified by observation of chest rise. In this analysis, the mean $\mathrm{V}_{\mathrm{T}}$ for the Upright was significantly higher when no chest rise was seen. However, upon further analysis of mean $\mathrm{V}_{\mathrm{T}}$ for individual sequences, all comparisons for the Upright were not significant: sequence 1 (ventilation with low compliance), $P=.26$; sequence 2 (ventilation with normal compliance),
$P=.07$; sequence $3, P=.18$; sequence $4, P=.10$; and sequences 1 and 4 together, $P=.09$ (data not shown).

Data for performance outcome variables for sequence 1 (low compliance for $1 \mathrm{~min}$ ) and sequence 4 (normal compliance for $2 \mathrm{~min}$ ) are displayed in Table 4. Low-compliance lung expansion $\left(0.5 \mathrm{~mL} / \mathrm{cm} \mathrm{H}_{2} \mathrm{O}\right)$ simulates newborns with fluid-filled lungs, and normal-compliance lung expansion $\left(2.0 \mathrm{~mL} / \mathrm{cm} \mathrm{H}_{2} \mathrm{O}\right)$ simulates infants with fully expanded lungs.

For the low-compliance lung scenario (sequence 1), the performance results were generally slightly higher for the Upright than for the NeoNatalie. The percentage of inadequate ventilations was significantly lower for the Upright than for the NeoNatalie with both low compliance $(8.31 \%$ vs $19.01 \%$ ) and normal compliance ( $1.05 \%$ vs $8.64 \%)$. For the normal-compliance lung scenario (sequence 4), the results showed a high percentage of excessive ventilations for both the Upright and NeoNatalie (85.92\% vs $71.39 \%)$.

\section{Disassembly/Reassembly}

Data from observation of participants disassembling and reassembling both devices are shown in Table 5. Participants were able to correctly disassemble all parts of the NeoNatalie device less often than those of the Upright device (summary index score of $36 \%$ vs $79 \%$ ). Disassembly of the NeoNatalie device took longer $(87.9 \pm 44.2 \mathrm{~s})$ compared with the Upright device $(64.3 \pm 39.1 \mathrm{~s})(P=.02)$. Participants were able to correctly assemble all parts of the NeoNatalie device less often than those of the Upright device (summary index score of $13 \%$ vs $53 \%$ ). Reassembly of the NeoNatalie device took longer $(166.03 \pm 25.02 \mathrm{~s})$ compared with the Upright device $(128 \pm 44.3 \mathrm{~s})(P<.001)$. In terms of acceptability, participants considered both disassembly and reassembly of the Upright to be significantly easier compared with the NeoNatalie.

In debriefing interviews, many participants noted that fewer parts and the instructions on the Upright device made disassembly easier. Generally, participants stated that the larger number of parts of the NeoNatalie device and the absence of instructions made it much more difficult to reassemble the device. Participants reported that the intake valve was difficult to reassemble in both devices and that it was particularly difficult to get it to lay flat once in place. One participant summed up the device differences by referring to the NeoNatalie device as the Microsoft product and the Upright device as the Apple product.

Disassembly and reassembly data were analyzed by user experience and hand size. The summary disassembly and summary reassembly indices were significantly higher for the Upright device across all groups, and the differences in mean times of disassembly and reassembly were not significantly different across any group (data not shown). 


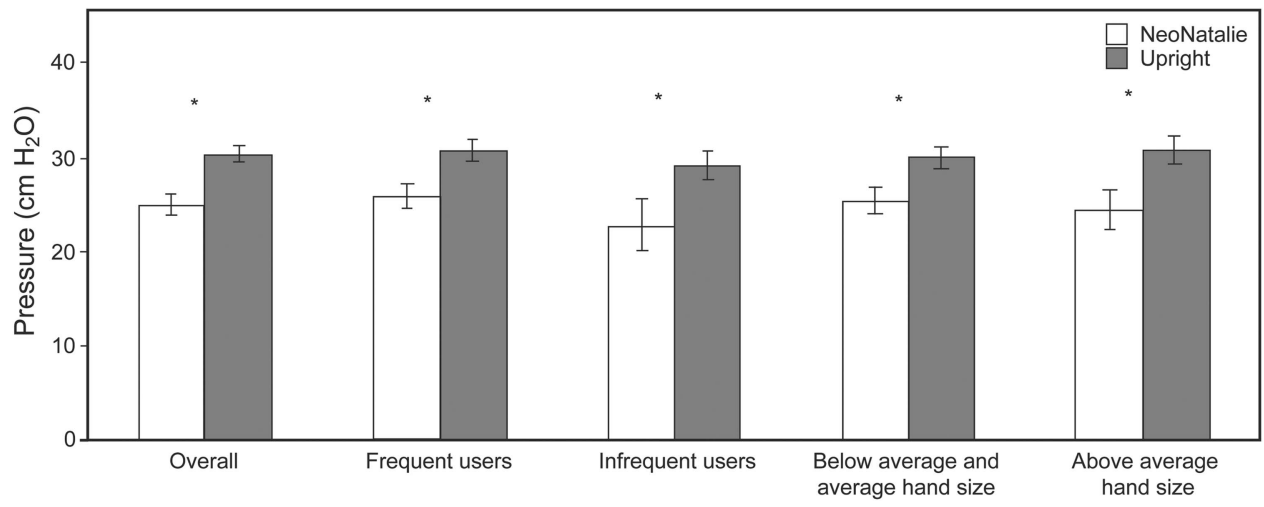

Fig. 2. Lung model pressure for a 4-min testing epoch. Values represent mean \pm SEM for each manual resuscitator. ${ }^{*} P<.05$.

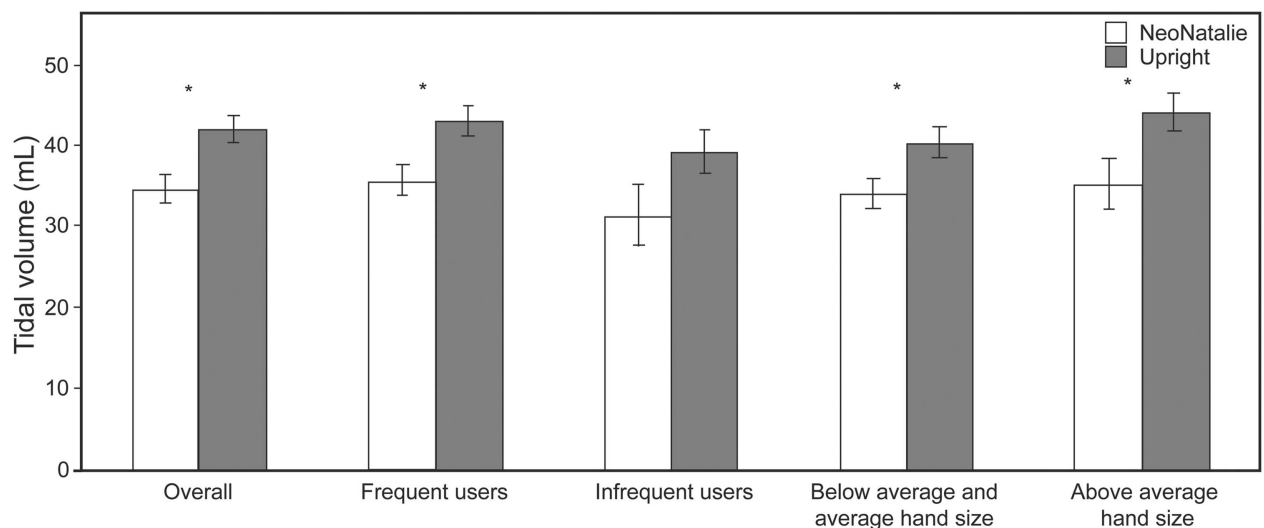

Fig. 3. Lung model tidal volume for a 4-min testing epoch. Values represent mean \pm SEM for each manual resuscitator. ${ }^{*} P<.05$.

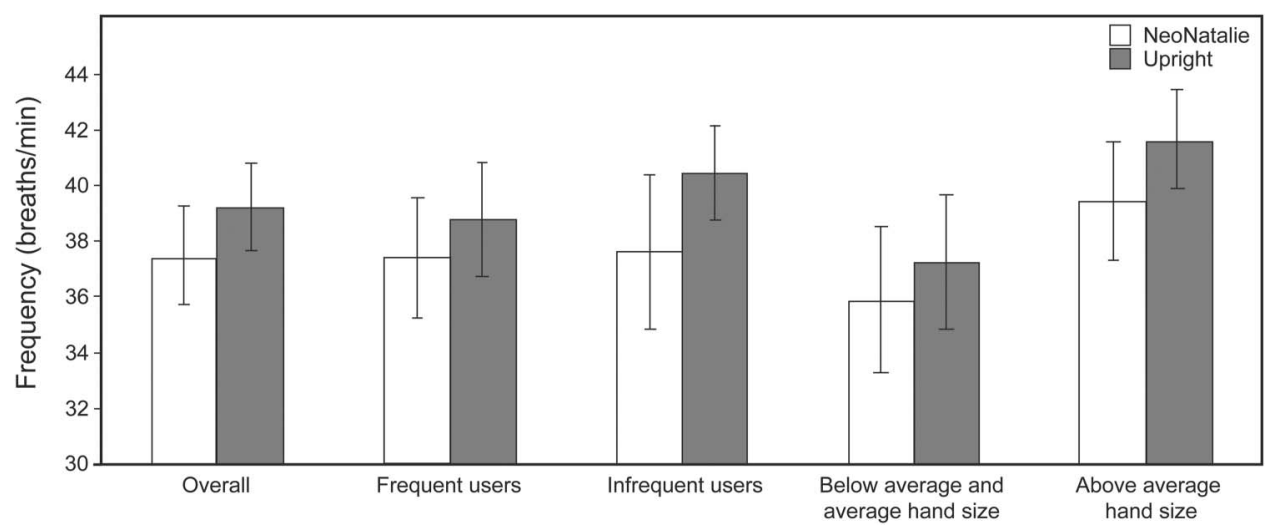

Fig. 4. Lung model breathing frequency for a 4-min testing epoch. Values represent mean \pm SEM for each manual resuscitator.

\section{Acceptability}

The majority (68\%) of participants stated that they preferred the Upright resuscitator. Interestingly, neither user experience nor hand size affected their stated preference (data not shown). Of the 15 acceptability variables included in the user acceptability index (Table 6), 3 (apparent durability, ease of holding the mask, and general ease of use) indicated significantly higher acceptability for the Upright resuscitator. One variable (ability to observe chest rise) showed significantly higher acceptability for the NeoNatalie device. The summary acceptability score indicated a significantly higher acceptability across all 15 variables for the Upright device.

In debriefing interviews, participants noted that the Upright resuscitator bag felt more solid, sturdier, and hardier. 
Table 3. Performance Outcome Variables for Both Devices During a 4-Minute Ventilation Sequence

\begin{tabular}{|c|c|c|c|}
\hline Variable & NeoNatalie & Upright & $P$ \\
\hline Mean $\mathrm{V}_{\mathrm{T}}, \mathrm{mL}$ & 34.3 & 42.1 & $<.001 *$ \\
\hline Inadequate $\mathrm{V}_{\mathrm{T}}(<5 \mathrm{~mL} / \mathrm{kg}, 12.5 \mathrm{~mL}), \% \dagger$ & 14.4 & 6.2 & $<.001 *$ \\
\hline Excessive $\mathrm{V}_{\mathrm{T}}(>15 \mathrm{~mL} / \mathrm{kg}, 37.5 \mathrm{~mL}), \% \dagger$ & 37.4 & 43.9 & $<.001 *$ \\
\hline Mean PIP, $\mathrm{cm} \mathrm{H}_{2} \mathrm{O}$ & 25.0 & 30.4 & $<.001 *$ \\
\hline Excessive PIP, $\%$ over $60 \mathrm{~cm} \mathrm{H}_{2} \mathrm{O}$ & 0.19 & 0.44 & $<.020 *$ \\
\hline Mean minute volume, $\mathrm{mL} / \mathrm{min}$ & $1,120.7$ & $1,387.4$ & $<.001 *$ \\
\hline Mean breathing frequency, breaths/min & 38.25 & 40.24 & .16 \\
\hline Breathing frequency $<30$ breaths $/ \mathrm{min}, \%$ & 23.1 & 12.2 & $.01 *$ \\
\hline Breathing frequency $<40$ breaths $/ \mathrm{min}, \%$ & 60.5 & 58.1 & .67 \\
\hline Breathing frequency $>60$ breaths $/ \mathrm{min}, \%$ & 4.08 & 8.00 & .59 \\
\hline \multicolumn{4}{|c|}{$\begin{array}{l}* \text { Significant } P \text { values }(P<.05) \text {. Comparison of means between devices was determined using } \\
\text { a } t \text { test; comparison of categorical variables between devices was determined using a chi- } \\
\text { square test. } \\
\dagger \text { Based on } 2.5 \text {-kg infant weight. } \\
\mathrm{V}_{\mathrm{T}}=\text { tidal volume } \\
\mathrm{PIP}=\text { peak inspiratory pressure }\end{array}$} \\
\hline
\end{tabular}

Generally, participants stated that they liked the Upright mask because it was bigger and thereby provided more surface to hang on to, the shape allowed for a more secure hold, and it was more comfortable to grip. Many participants noted that the orientation of the bag to the mask stem on the Upright resuscitator seemed intuitive and was relatively easy to use. Some participants stated that the Upright design was more tiring than the NeoNatalie device, noting that the forearm was the part that tired rather than the hand holding the mask, as in the standard device.

Four different causes of fatigue during use were identified: (1) holding the bag in position (weight of device), (2) stiffness of bag material, (3) volume of bag (larger bag requires more exertion, but smallness of bag causes cramping of hand); and (4) holding the mask in position (maintaining seal). Nearly equal complaints about fatigue for both devices were recorded.

All participants stated that being able to see chest rise was a critical and necessary component of resuscitation. Many participants observed that the Upright device occluded the sight line for chest rise and found this to be a concern.

Acceptability data were analyzed by user experience and hand size. One variable (ease of reassembly) was significantly higher for the Upright device across all groups (data not shown). In addition, infrequent users rated the ease of occluding the pressure-relief valve to be higher for the Upright than for the NeoNatalie device. Frequent users rated the shape of the mask and ease of holding the mask to be significantly higher for the Upright than for the NeoNatalie device. Furthermore, the summary acceptability index for frequent users was higher for the Upright device. Participants with average and below-average hand size rated the apparent durability, ease of holding the mask, and ability to observe chest rise as being significantly better for the Upright device.

\section{Discussion}

The new Upright resuscitator was designed to be more user-friendly. The major finding of this study was that the Upright resuscitator may be more suitable for reducing ineffective manual ventilation during resuscitation by lessexperienced users. Although the most obvious feature is the upright orientation of the bag, there are, in fact, several other differences compared with the conventional NeoNatalie, some or all of which could have contributed to the differences in the results. The manufacturer has increased the bag size by $45 \%$ (from 220 to $320 \mathrm{~mL}$ ), thickened and flattened the mask dome, ensured better fitting into the mask stem, and changed the bag orientation to vertical and centered above the newborn's face from horizontal and adjacent to the face. The manufacturer intended the increased volume of the Upright device to compensate for leakages (ie, leakage from the device, mask/face interface, and air that is bled off from the resuscitator's built-in pressure-relief valve due to forceful ventilations), and the mask design with the thicker upper rim and vertical bag orientation makes it easier for the user to establish and maintain mask seal.

Inadequate ventilations can result in poor response to resuscitation. Hypoventilation is a major concern in asphyxiated babies and preterm infants with surfactant deficiency and fluid-filled lungs because more pressure is required to overcome resistive and inelastic components of the lung to provide adequate carbon dioxide clearance. Ventilations delivered with the Upright device had a significantly lower percentage of inadequate ventilations compared with the NeoNatalie in the low-compliance sequence $(8.31 \%$ vs $19.01 \%, P<.001)$ and in the normal-compliance sequence $(1.05 \%$ vs $8.64 \%, P<.001)$. These data suggest that use of the Upright resuscitator may be clinically relevant in addressing inadequate ventilations and may help in compensating for mask leaks.

When we assessed the entire 4-min testing epoch, the performance of the 2 designs differed significantly in $\mathrm{V}_{\mathrm{T}}$, pressure, and minute ventilation, with the Upright device delivering significantly more volume and pressure than the NeoNatalie device. The 4-min testing epoch was supposed to mimic a clinical scenario in which a newborn that has undergone adverse intrapartum events, such as birth asphyxia, would have stiff lungs with low compliance. This is why, initially, ventilation with prolonged inspiratory time is useful to open the alveoli and achieve normal compliance and adequate functional residual capacity. In fact, the Resuscitation Council in the United Kingdom advises beginning resuscitation initially with 5 inflation breaths lasting 2-3 s using pressures of up to $30 \mathrm{~cm} \mathrm{H}_{2} \mathrm{O}$ and 
Table 4. Performance Outcome Variables for Sequences 1 and 4

\begin{tabular}{|c|c|c|c|c|c|c|}
\hline \multirow{2}{*}{ Variable } & \multicolumn{3}{|c|}{ Sequence 1 (Low Compliance for $1 \mathrm{~min}$ ) } & \multicolumn{3}{|c|}{ Sequence 4 (Normal Compliance for $2 \mathrm{~min}$ ) } \\
\hline & NeoNatalie & Upright & $P$ & NeoNatalie & Upright & $P$ \\
\hline Mean $\mathrm{V}_{\mathrm{T}}, \mathrm{mL}$ & 24.7 & 27.8 & $<.001 *$ & 44.3 & 58.3 & $<.001^{*}$ \\
\hline $\mathrm{V}_{\mathrm{T}}<5 \mathrm{~mL} / \mathrm{kg}(12.5 \mathrm{~mL}), \% \dagger$ & 19.0 & 8.3 & $<.001 *$ & 8.6 & 1.1 & $<.001^{*}$ \\
\hline $\mathrm{V}_{\mathrm{T}}>15 \mathrm{~mL} / \mathrm{kg}(37.5 \mathrm{~mL}), \% \dagger$ & 4.9 & 5.1 & .84 & 71.4 & 85.9 & $<.001^{*}$ \\
\hline Mean PIP, $\mathrm{cm} \mathrm{H}_{2} \mathrm{O}$ & 28.9 & 33.6 & $<.001 *$ & 20.8 & 27.0 & $<.001^{*}$ \\
\hline Excessive PIP, $\%$ over $60 \mathrm{~cm} \mathrm{H}_{2} \mathrm{O}$ & 0.72 & 0.59 & .66 & 0 & 0.14 & .047 \\
\hline Mean minute volume, $\mathrm{mL} / \mathrm{min}$ & $1,022.0$ & $1,265.8$ & $.02 *$ & $1,692.1$ & $2,250.5$ & $.001 *$ \\
\hline Mean breathing frequency, breaths/min & 41.4 & 45.5 & .17 & 38.2 & 38.6 & .81 \\
\hline Breathing frequency $<30$ breaths $/ \mathrm{min}, \%$ & 13.5 & 8.1 & .45 & 16.2 & 10.8 & .49 \\
\hline Breathing frequency $<40$ breaths $/ \mathrm{min}, \%$ & 43.2 & 35.1 & .47 & 64.9 & 54.1 & .34 \\
\hline Breathing frequency $>60$ breaths $/ \mathrm{min}, \%$ & 8.1 & 10.8 & 69 & 0 & 0 & NA \\
\hline \multicolumn{7}{|c|}{$\begin{array}{l}\text { * Significant } P \text { values }(P<.05) \text {. Comparison of means between sequences was determined using a } t \text { test; Comparison of categorical variables between sequences was determined using a chi-square } \\
\text { test. } \\
\dagger \text { Based on } 2.5 \text {-kg infant weight. } \\
\mathrm{V}_{\mathrm{T}}=\text { tidal volume } \\
\text { PIP }=\text { peak inspiratory pressure } \\
\mathrm{NA}=\text { not applicable }\end{array}$} \\
\hline
\end{tabular}

Table 5. Device Disassembly and Reassembly

\begin{tabular}{|c|c|c|c|c|c|c|}
\hline \multirow{3}{*}{ Part } & \multicolumn{6}{|c|}{ Correct Placement of Each Part, $n(\%)$} \\
\hline & \multicolumn{3}{|c|}{ Disassembly } & \multicolumn{3}{|c|}{ Reassembly } \\
\hline & NeoNatalie & Upright & $P$ & NeoNatalie & Upright & $P$ \\
\hline Duckbill valve & $31(94)$ & $33(100)$ & (Constant) & $21(66)^{*}$ & $30(97) \dagger$ & .32 \\
\hline Intake valve housing & $29(88)$ & $30(91)$ & .33 & $17(53)^{*}$ & $28(88)^{*}$ & $>.99$ \\
\hline Bag & $31(94)$ & $33(100)$ & (Constant) & $20(63)^{*}$ & $29(94) \dagger$ & $>.99$ \\
\hline Intake valve & $26(79)$ & $30(91)$ & .52 & $12(38)^{*}$ & $20(63)^{*}$ & $.01 \ddagger$ \\
\hline Pressure-relief valve & $28(85)$ & $29(88)$ & .09 & $25(78)^{*}$ & $27(87) \dagger$ & $>.99$ \\
\hline Mask & $33(100)$ & $33(100)$ & (Constant) & $23(74) \dagger$ & $30(97) \dagger$ & $>.99$ \\
\hline Mask housing & $30(94)^{*}$ & $33(100)$ & (Constant) & $25(78)^{*}$ & $30(97) \dagger$ & $>.99$ \\
\hline Exhalation disk membrane & $33(100)$ & NA & NA & $9(28)^{*}$ & NA & NA \\
\hline Elbow joint & $16(48)$ & NA & NA & $19(59)^{*}$ & NA & NA \\
\hline Oxygen connection housing & $30(91)$ & NA & NA & $16(50)^{*}$ & NA & NA \\
\hline Summary disassembly index & $12(36)$ & $26(79)$ & $>.99$ & NA & NA & NA \\
\hline Summary reassembly index & NA & NA & NA & $4(13) \dagger$ & $17(53)^{*}$ & .10 \\
\hline \multicolumn{7}{|c|}{$\begin{array}{l}N=33 . \\
* \text { Data were missing for one participant }(n=32) \text {. } \\
\dagger \text { Data were missing for } 2 \text { participants }(n=31) \text {. } \\
\ddagger P<.05 \text { using the Fisher exact test (2-sided). } \\
\text { NA }=\text { not applicable }\end{array}$} \\
\hline
\end{tabular}

thereafter $30-40$ breaths/min as required. ${ }^{13}$ The Dutch neonatal resuscitation guidelines also recommend the use of 5 initial inflations of 2-3 s in both term and preterm babies. ${ }^{14}$ Although this type of test scenario may be useful in assessing providers from a real-life clinical perspective, it may not necessarily be the most helpful in assessing device performance. For this reason, we independently analyzed performance for low- and normal-compliance test sequences. The test sequences with low-compliance lung settings showed no difference in the percentage of exces- sive ventilations $(>37.5 \mathrm{~mL})$ between the Upright and NeoNatalie devices $(5.05 \%$ vs $4.90 \%, P=.85)$. On the other hand, the test sequences with normal-compliance lung settings showed that ventilations delivered with both the NeoNatalie and Upright resuscitators yielded a higher percentage of excessive ventilations $(>37.5 \mathrm{~mL})$. This increase was greater with the Upright device than with the NeoNatalie $(85.92 \%$ vs $71.39 \%, P<.001)$.

Preterm infants are at risk for lung injury because of structurally immature lungs, absence of a stiff chest wall, 
Table 6. Acceptability of Devices

\begin{tabular}{|c|c|c|c|}
\hline \multirow{2}{*}{ Features } & \multicolumn{3}{|c|}{ Device Acceptability (mean $\pm \mathrm{SD}$ ) } \\
\hline & NeoNatalie & Upright & $P$ \\
\hline Apparent durability & $4.24 \pm 0.76^{*}$ & $4.45 \pm 0.56$ & $.044 \dagger$ \\
\hline Size of bag & $4.26 \pm 0.76$ & $4.13 \pm 0.78$ & .44 \\
\hline Orientation of bag to mask stem & $4.11 \pm 0.80$ & $4.24 \pm 0.83 *$ & .50 \\
\hline Size of mask & $3.73 \pm 0.90 *$ & $4.05 \pm 0.84$ & .08 \\
\hline Shape of mask & $3.67 \pm 0.83$ & $3.87 \pm 0.94$ & .08 \\
\hline Feel of material & $4.03 \pm 0.69 *$ & $4.26 \pm 0.83$ & .08 \\
\hline Ease of holding mask & $3.34 \pm 0.88$ & $4.08 \pm 0.94$ & $<.001$ \\
\hline Ease of holding bag & $3.97 \pm 0.68$ & $4.00 \pm 0.99$ & .89 \\
\hline Comfort during use & $3.68 \pm 0.70$ & $3.97 \pm 0.96^{*}$ & .16 \\
\hline Ease of use (general) & $3.79 \pm 0.70$ & $4.11 \pm 0.89$ & $.044 \dagger$ \\
\hline Ease of ventilation & $3.61 \pm 0.82$ & $3.79 \pm 0.99$ & .36 \\
\hline Ease of rate & $4.05 \pm 0.70$ & $4.03 \pm 0.79$ & .80 \\
\hline Ability to observe chest rise & $4.16 \pm 0.92$ & $3.74 \pm 1.08$ & $.044 \dagger$ \\
\hline Ease of occluding pressure-relief valve & $3.37 \pm 1.00$ & $3.58 \pm 1.13$ & .23 \\
\hline Fatigue during use & $3.63 \pm 0.88$ & $3.50 \pm 0.83$ & .45 \\
\hline Summary acceptability index $(n=33)$ & $58.27 \pm 6.59$ & $61.12 \pm 9.17$ & $.041 \dagger$ \\
\hline Ease of disassembly $(n=33)$ & $3.21 \pm 0.99$ & $3.73 \pm 1.01$ & $.039 \dagger$ \\
\hline Ease of assembly $(n=31)$ & $1.97 \pm 0.88$ & $3.65 \pm 1.08$ & $<.001$ \\
\hline
\end{tabular}

and surfactant deficiency. ${ }^{15}$ There is compelling evidence that short-term exposure to excessive $\mathrm{V}_{\mathrm{T}}$ during mechanical ventilation initiates lung inflammation in premature infants, even after only a few minutes of manual resuscitation. ${ }^{16}$ Ventilation for $15 \mathrm{~min}$ with a $\mathrm{V}_{\mathrm{T}}$ of $15 \mathrm{~mL} / \mathrm{kg}$ has been shown to cause an injurious lung process in infant animal studies. ${ }^{17}$ As few as 3 over-distending breaths at birth have been shown to compromise the therapeutic effect of subsequent surfactant replacement in an animal model of prematurity. ${ }^{18}$ Conversely, $\mathrm{V}_{\mathrm{T}}$ ventilation of $<5 \mathrm{~mL} / \mathrm{kg}$ has been associated with inflammatory response in the lungs of babies receiving mechanical ventilation. ${ }^{19}$ Our test lung, mannikin, and chest-rise module were configured for a term newborn, not a low-birthweight neonate. It is unclear how excessive or inadequate $V_{T}$ could impact the lungs of a term newborn with regard to injury. Although we cannot extrapolate from the aforementioned data obtained from premature subjects, we speculate that stiff or poorly compliant lungs are more susceptible to injury, regardless of the gestational age. Based on our findings, there were no differences in the percentage of excessive ventilations $(>37.5 \mathrm{~mL}$ ) between the Upright and NeoNatalie devices during ventilation at the low-compliance lung settings. The percentage of excessive ventilations $(71.39 \%$ ) with the NeoNatalie, even with its smaller bag, was well above what would be expected from the participants. It is possible that the results are partly due to the testing sequence that was not randomized and did not pause between the low- and normal-compliance sequences. Participants had initially provided sustained and augmented ventilations to achieve chest rise on the mannikin when the test was set for low compliance. This method of ventilation could have continued even when the compliance setting was made normal, resulting in over-ventilation. Changes in compliance are not always clinically easy to perceive. ${ }^{20-22}$ The greater difference with the Upright device could also be partly due to the larger bag size in addition to the improved mask design. Avoiding excessive $\mathrm{V}_{\mathrm{T}}$ delivery could be achieved during manual ventilation with a volume or pressure monitor, ${ }^{23}$ but these devices are unlikely to be available in resource-limited settings. As such, training focused on limiting excessive chest rise is of the utmost importance in these settings.

Interestingly, although there were variations in the PIP, it did not demonstrate the excessive rise observed with $\mathrm{V}_{\mathrm{T}}$. This was probably due to the protective pressure-relief valves. Although the excessive pressures were more common with the Upright, it is interesting that they were also observed with the NeoNatalie, which also has a preset pressure-relief valve. The higher pressures noted with the Upright device could have been due to the higher pressure limit set for the pressure-relief valve. Some clinicians disabled the pressure-relief valves with the NeoNatalie to provide better chest rise, as would be done in the clinical 
setting. It was not clear whether this was done because they were more familiar with the location of the pressurerelief valve in the NeoNatalie than with the Upright or if the latter was more difficult to occlude. This study also highlights clearly that although pressure-relief valves can protect against the development of excessive PIPs, they obviously do not protect against the administration of excessive $\mathrm{V}_{\mathrm{T}}$. $\mathrm{V}_{\mathrm{T}}$ is even more critical in damaging the alveoli, especially in preterm babies. ${ }^{24}$ Innovations that can detect and safeguard against excessive $\mathrm{V}_{\mathrm{T}}$ can indeed be very useful.

Although a variety of clinical parameters have been used as diagnostic features of adequate ventilation, it is now being realized that some of these, such as heart rate, respiration, and chest excursion, have their own limitations. ${ }^{25,26}$ In practical terms, whether training on a mannikin or during resuscitation of a baby, chest rise is an important component; health workers are encouraged to produce an obvious chest rise. This is despite the fact that chest movements in sleeping babies with normal $\mathrm{V}_{\mathrm{T}}$ are barely visible. ${ }^{23}$ In our study, the inability of some of the participants to visualize chest rise due to equipment malfunction did present a challenge but also served as an unplanned learning experience. Participants were able to ventilate and obtain similar pressures and $\mathrm{V}_{\mathrm{T}}$ whether they saw chest rise. The results of this study suggest that participants relied on the subjective feel of resistance related to changes in lung mechanics when they could not visualize the chest rise. The inability to observe the chest rise could have contributed to the mean $\mathrm{V}_{\mathrm{T}}$ for the Upright being significantly higher when no chest rise was seen. However, as noted, upon further analysis of mean $\mathrm{V}_{\mathrm{T}}$ for individual sequences, all comparisons for the Upright were not significant.

Changes in lung compliance take place after birth, especially in preterm infants and babies with birth asphyxia. Unfortunately, however, health workers do not always readily feel changes in compliances. ${ }^{26}$ It is also possible that aiming for obvious chest rise may not always be appropriate, especially after the initial ventilations during the period of low compliance, and more so in preterm babies as there may be risk of high $\mathrm{V}_{\mathrm{T}}$. However, it may be difficult to formulate suitable guidelines, as there are currently no objective criteria to document the degree of chest rise rise/expansion in mannikins, let alone in babies. Chest rise as a subjective measure of device performance should be explored further, especially to determine more objective methods of documentation, including relationship to parameters such as $\mathrm{V}_{\mathrm{T}}$.

Regarding acceptability, participants found the Upright device to be better overall across a variety of distinct measures regardless of user frequency or hand size. Likewise, users were able to disassemble and assemble the Upright device correctly more often than the NeoNatalie device regardless of user frequency or hand size. The lack of significant differences in disassembly/reassembly mean times in both groups indicated that user experience and hand size did not affect procedure length. One issue noted was that a direct view of the infant's chest was restricted by the Upright design, more so when ventilation was carried out seated, and users may need to shift their head to the side to observe the chest.

The most important issue may be degree of skill influencing the quality of care rendered. This can be significantly influenced by improved competency-based training and follow-up supportive mentoring and supervision. Training programs may need to emphasize correct application and fixation of the mask, prevention of mask leak, and proper ventilation technique. In addition, although initial ventilations often need to be somewhat stronger with prolonged inspiration, care has to be taken to avoid excessive ventilation, especially in the subsequent period. Particularly in the latter phase as resuscitation progresses, perhaps the recommendation for an obvious chest rise may need to be tempered to a milder chest rise.

This in vitro study has certain limitations. We used only one lung model configuration and settings that are commonly used. We did not test the range of settings that are commonly used. It is possible that different support settings and lung-mechanics configurations could render different results. More research is required to develop simple, innovative measures to document $V_{T}$ and safeguards that avoid excessive $\mathrm{V}_{\mathrm{T}}$ just as the pressure-relief valve limits the PIP.

\section{Conclusions}

The performance and acceptability of the Upright device in this user population suggest that the device may be suitable for infrequent users in low-resource settings; the Upright device should be tested in such a setting. $\mathrm{V}_{\mathrm{T}}$ delivery to infant lungs is extremely important, and development of low-cost innovations to safeguard against excess, such as in the case of pressure-relief valves that protect against excessive pressure, would be beneficial.

\section{ACKNOWLEDGMENTS}

We thank Greg Kachmarik for excellent engineering support, Pooja Bansil for superb data analysis, and Jillian Zemanek and Abra Greene for cheerful efforts to support the implementation of the study and preparation of this manuscript.

\section{REFERENCES}

1. Liu L, Johnson HL, Cousens S, Perin J, Scott S, Lawn JE, et al. Global, regional, and national causes of child mortality: an updated systematic analysis for 2010 with time trends since 2000. Lancet 2012;379(9832):2151-2161.

2. Bhutta ZA, Yakoob MY, Lawn JE, Rizvi A, Friberg IK, Weissman E, et al. Stillbirths: what difference can we make and at what cost? Lancet 2011;377(9776):1523-1538. 
3. World Health Organization. Guidelines on basic newborn resuscitation. Geneva, Switzerland: WHO Press; 2012.

4. Bennett S, Finer NN, Rich W, Vaucher Y. A comparison of three neonatal resuscitation devices. Resuscitation 2005;67(1):113-118.

5. O'Donnell CP, Gibson AT, Davis PG. Pinching, electrocution, ravens' beaks, and positive pressure ventilation: a brief history of neonatal resuscitation. Arch Dis Child Fetal Neonatal Ed 2006;91(5):F369-F673.

6. ISO 10651-4:2002(E). Lung ventilators-part 4: particular requirements for operator-powered resuscitators. Geneva, Switzerland: International Organization for Standardization; 2002.

7. Ersdal HL, Mduma E, Svensen E, Perlman JM. Early initiation of basic resuscitation interventions including face mask ventilation may reduce birth asphyxia related mortality in low-income countries: a prospective descriptive observational study. Resuscitation 2012; 83(7):869-873.

8. DiBlasi RM, Richardson CP, Hansen T. Pulmonary physiology of the newborn. In: Gleason A, Devasker S, editors. Avery's diseases of the newborn, 9th edition. Philadelphia, PA: Saunders; 2012;598-611.

9. Wyllie J, Perlman JM, Kattwinkel J, Atkins DL, Chameides L, Goldsmith JP, et al. Part 11: neonatal resuscitation: 2010 International Consensus on Cardiopulmonary Resuscitation and Emergency Cardiovascular Care Science with Treatment Recommendations. Resuscitation 2010;81(Suppl 1):e260-2287.

10. Vyas H, Milner AD, Hopkin IE, Boon AW. Physiologic responses to prolonged and slow-rise inflation in the resuscitation of the asphyxiated newborn infant. J Pediatr 1981;99(4):635-639.

11. Boon AW, Milner AD, Hopkin IE. Lung expansion, tidal exchange, and formation of the functional residual capacity during resuscitation of asphyxiated neonates. J Pediatr 1979;95(6):1031-1036.

12. Lindner W, Vossbeck S, Hummler H, Pohlandt F. Delivery room management of extremely low birth weight infants: spontaneous breathing or intubation? Pediatrics 1999;103(5 Pt 1):961-967.

13. Resuscitation Council (UK). Newborn life support: resuscitation at birth, 2nd edition. London, UK: Resuscitation Council; 2006.

14. Hooper SB, Te Pas AB, Lewis RA, Morley CJ. Establishing functional residual capacity at birth. NeoReviews 2010;11(9):e474-e483.

15. Schmölzer GM, Te Pas AB, Davis PG, Morley CJ. Reducing lung injury during neonatal resuscitation of preterm infants. J Pediatr 2008;153(6):741-745.
16. Nilsson R, Grossmann G, Robertson B. Lung surfactant and the pathogenesis of neonatal bronchiolar lesions induced by artificial ventilation. Pediatr Res 1978;12(4 Pt 1):249-255.

17. Hillman NH, Moss TJ, Kallapur SG, Bachurski C, Pillow JJ, Polglase GR, et al. Brief, large tidal volume ventilation initiates lung injury and a systemic response in fetal sheep. Am J Respir Crit Care Med 2007;176(6):575-581

18. Björklund LJ, Ingimarsson J, Curstedt T, John J, Robertson B, Werner O, Vilstrup CT. Manual ventilation with a few large breaths at birth compromises the therapeutic effect of subsequent surfactant replacement in immature lambs. Pediatr Res 1997;42(3):348-355.

19. Lista G, Castoldi F, Fontana P, Reali R, Reggiani A, Bianchi S, Compagnoni G. Lung inflammation in preterm infants with respiratory distress syndrome: effects of ventilation with different tidal volumes. Pediatr Pulmonol 2006;41(4):357-363.

20. Spears RS Jr, Yeh A, Fisher DM, Zwass MS. The "educated hand." Can anesthesiologists assess changes in neonatal pulmonary compliance manually? Anesthesiology 1991;75(4):693-696.

21. Heneghan C. "The educated hand." Anesthesiology 1992;76(6):1063.

22. Bowman TA, Paget-Brown A, Carroll J, Gurka MJ, Kattwinkel J. Sensing and responding to compliance changes during manual ventilation using a lung model: can we teach healthcare providers to improve? J Pediatr 2012;160(3):372.e1-376.e1.

23. Kattwinkel J, Stewart C, Walsh B, Gurka M, Paget-Brown A. Responding to compliance changes in a lung model during manual ventilation: perhaps volume, rather than pressure, should be displayed. Pediatrics 2009;123(3):e465-e470.

24. Kamlin CO, O’Donnell CP, Everest NJ, Davis PG, Morley CJ. Accuracy of clinical assessment of infant heart rate in the delivery room. Resuscitation 2006;71(3):319-321.

25. Schmölzer GM, Kamlin OC, O’Donnell CP, Dawson JA, Morley CJ, Davis PG. Assessment of tidal volume and gas leak during mask ventilation of preterm infants in the delivery room. Arch Dis Child Fetal Neonatal Ed 2010;95(6):F393-F397.

26. Brugada M, Schilleman K, Witlox RS, Walther FJ, Vento M, Te Pas $\mathrm{AB}$. Variability in the assessment of 'adequate' chest excursion during simulated neonatal resuscitation. Neonatology 2011;100(1): 99-104.

This article is approved for Continuing Respiratory Care Education credit. For information and to obtain your CRCE

(free to AARC members) visit

www.rcjournal.com

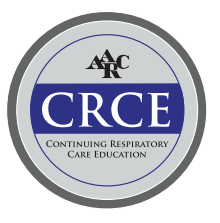

